\title{
ANÁlISIS DE CICLO DE VIDA PARA LA PRODUCCIÓN DE BIODIESEL DERIVADO DE PALMA DE ACEITE, CASO COLOMBIANO
}

\author{
Life Cycle Analysis for biodiesel production \\ FROM PALM OIL CASE COLOMBIAN
}

\author{
${ }^{1}$ Julio Mario Franco Rodríguez, ${ }^{2}$ Luis Hernán Ordońez Noriega, \\ ${ }^{3}$ Israel Herrera Orozco ${ }^{4}$ Jesús Alfonso Torres Ortega \\ ${ }^{1}$ Facultad de Ingenieria, Universidad de La Salle, Bogotá, Colombia \\ ${ }^{2}$ Facultad de Ingenieria, Universidad de La Salle, Bogotá, Colombia. \\ ${ }^{3}$ Unidad de Análisis de Sistemas Energéticos. CIEMAT. Madrid, España. \\ ${ }^{4}$ Facultad de Ingenieria, Centro Lasallista de Investigación y Modelación Ambiental - \\ CLIMA, Universidad de La Salle, Bogotá, Colombia.
}

\section{RESUMEN}

La producción de biodiesel a partir de aceite vegetal derivado de palma africana, requiere diferentes procesos, los cuales podrían provocar daños ambientales, como las emisiones atmosféricas, la acidificación, la desertificación y el vertido de aguas residuales, que contribuyen al cambio climático. En este trabajo se realiza el análisis del ciclo de vida (ACV) para la producción de biodiesel a partir de aceite vegetal, utilizando la metodología de la norma técnica ISO 14044, para lo cual fue necesario realizar un análisis de inventario, considerando las materias primas y la energía para cada proceso. El análisis del ciclo de vida "de la cuna a la puerta”, busca la evaluación de impactos ambientales mediante el uso del software SimaPro permitiendo identificar las fases con mayor impacto, además se generan recomendaciones para optimizar la cadena de producción y mitigación de las cargas medioambientales relevantes. Según la evaluación del ciclo de vida usando la metodología ISO 14044 para las diferentes categorías de impacto que se evaluaron, se concluye que la actividad con el mayor impacto ambiental es la extracción de aceite de la palma. Hay cargas favorables para el medio ambiente que se producen por la recirculación de insumos como el metanol; asimismo, en la fase de reacción, se generó glicerina, este subproducto se utiliza en la industria farmacéutica.

Palabras clave: aceite de palma, ACV, impactos, ISO 14044, SimaPro.

\footnotetext{
1 jfranco80@unisalle.edu.co, orcid.org/0000-0001.6498.0827

2 lordonez97@unisalle.edu.co

3 israel.herreralciemat.es, orcid.org/0000-0003-1864152x

4 jatorres @unisalle.edu.co, orcid.org/0000-0002-4476-0816
} 


\section{Abstract}

The production of biodiesel from vegetable oil derived of African oil palm requires different activities. All this could leads to environmental damage such as atmospheric emissions, acidification, desertification, dumping of wastewater, which contributes to climate change. In this work, the life cycle analysis (LCA) applied for the pro-duction of biodiesel from vegetable oil, using the methodology of the ISO 14044 technical standard, for which it was necessary to make an inventory analysis where the mass and energy flows at the entrance and outs of each phase of the production line. Researhing the life cycle analysis "cradle to gate", for the evaluation of environmental impacts troght the use of SimaPro 8.1 software where the phases with the greatest impact on the en-vironment are identified, which allows making recommendations to optimize the chain of production and to mitigate the relevant environmental burdens. According to the evaluation, methodology of the SimaPro software, and the different impact categories that were evaluate is concluding that the activity with the greatest environmental impact is the extraction of oil from African palm. There are loads favorable to the environment that are produced by the recirculation of inputs such as methanol, likewise in the reaction phase, glycerin was generated, this by-product is used in the pharmaceutical industry, thus avoiding a waste.

Keywords: Impacts, ISO 14044, LCA, Palm oil, SimaPro.

\section{INTRODUCCIÓN}

En la actualidad se han desarrollado tecnologías con el fin de contribuir en los estudios de mitigación de los impactos relacionados con las emisiones atmosféricas ocasionadas por la utilización de combustibles fósiles, $\mathrm{y}$, con la actividad industrial derivada de su uso, dado que esta actividad es una de las que más efectos adversos causan al ambiente, como la emisión de sustancias impactantes de particulados y residuos no degradables (Torres et al., 2018). Frente a los altos precios de los hidrocarburos y a su incidencia en el calentamiento global, la mayor parte de los países del mundo han venido promoviendo como alternativa la energía limpia y renovable, frente al petróleo, al gas y al carbón. Lo anterior nos lleva a tomar medidas con las que podamos ofrecer un entorno más amigable a través de tecnologías basadas en cuantificar los recursos energéticos y caracterizar las materias primas de cualquier proceso biológico, tecnológico o industrial, en la búsqueda de una sostenibilidad de los recursos privilegiando la reducción de los residuos generados o encontrando un valor agregado para estos (Torres et al., 2009).

Colombia ha venido desarrollado políticas para la introducción de biocombustibles como mezclas en la gasolina (nafta) y en el diésel mediante normas que obligan a agregar una mezcla gradualmente para el territorio nacional, y que podrá impulsar la producción de cultivos agroenergéticos para la generación de alcohol carburante y biodiesel (Ministerio de Minas y Energía 2018). Sin embargo, la propuesta de utilizar etanol derivado de la caña de azúcar para ser mezclado como carburante en la gasolina y así aumentar el rendimiento y disminuir la emisión de gases contaminantes puede traer inconvenientes diversos, inclusive para la seguridad alimentaria, aunque la principal preocupación es que se puede comprometer la oferta de azúcar y generar insatisfacción entre los consumidores, causando una inestabilidad en el precio del mercado. Es claro que estos efectos deben ser evaluados con profundidad para que se puedan tomar las medidas adecuadas. Entre las ventajas de la utilización del etanol está la disminución de emisiones vehiculares de nitrógeno en un 22 $\%$, de monóxido de carbono en un $50 \%$ y de hidrocarburos en un $3 \%$, además de ser una alternativa a la dependencia de combustibles de origen fósil (Arango $\&$ Torres, 2008).

Por otro lado, teniendo en cuenta que Colombia se encuentra entre los principales países productores de aceite de palma, con un área sembrada de $452 \mathrm{mil}$ 
hectáreas y más de un millón de toneladas al año, y que se consolidó como el primer país latinoamericano, y el quinto en el mundo en la producción de aceite de palma, ha llevado a mirar el sector palmicultor como una alternativa amigable con el medioambiente, por cuanto el biodiesel producido puede llegar a menos de 15 ppm de azufre, lo cual ha sido considerado para que las nuevas flotas de transporte utilicen mezclas de combustible diésel con al menos $10 \%$ de biodiésel (B10) y la meta es alcanzar un $100 \%$ como se ha logrado en otros países (Huergo 2018). El ACV plantea manejar los residuos en una forma sustentable desde el punto de vista ambiental minimizando todos los impactos asociados con el sistema de manejo (McDonough \& Braungart 2002).

En el presente trabajo se identifica y cuantifica las cargas ambientales generadas en el proceso de transformación del aceite de palma para la obtención de biodiesel, empezando con el cultivo y extracción del aceite mediante el análisis del ciclo de vida. La metodología empleada es la indicada en la Norma ISO 14044 para el diagnóstico y elaboración del inventario de datos, y se utilizó el software SimaPro 8.1 con el fin de determinar los impactos. La Norma permite establecer una guía para las etapas que se deben tener encuenta al momento del estudio con el objeto de desterminar un Análisis de Ciclo de Vida (ACV) y con el software se analiza la información obtenida para posteriormente identificar los impactos ambientales generados en cada etapa del procesamiento del aceite de palma, para con base en esto, formular las alternativas que conllevan a la prevención y/o minimización de los impactos sin que se afecte la calidad del producto.

\section{Metodología}

La metodología está enfocada en dos aspectos relevantes, primero, la identificación de los procesos involucrados en la obtención del aceite y su posterior procesamiento hasta biodiesel y segundo, la valoración de las cargas ambientales mediante el uso del software SimaPro. Para la evaluación de procesos involucrados en la producción de B10 fue necesario acudir a las bases de datos comerciales (Ecoinvent), también se recurrió al apoyo de datos bibliográficos de información relacionada con el fin de complementar la información relevante de cada proceso. La valoración ambiental está sustentada bajo la metodología sugerida por la norma técnica internacional ISO 14044 que abarca los principios y marco de referencia para el ACV, y a su vez, el análisis de cargas ambientales se realizó con el software o herramienta informática SimaPro de reconocido uso en estudios científicos.

Para ejecutar un análisis de ciclo de vida (ACV), es esencial contar con datos de todas las materias primas usadas, flujos de entradas y salidas de cada proceso con el fin de tener una fuente de resultados más completa y confiable, así mismo se deben tener en cuenta los límites o alcances de los sistemas. Por lo anterior, para cumplir con el objetivo del estudio, se inició con la recolección de los datos vinculados en los procesos de obtención del aceite de palma y su posterior transformación a biodiesel, luego se realizaron los cálculos de materias primas necesarias para alcanzar un kilómetro de recorrido de un bus articulado utilizado en la ciudad de Bogotá (Transmilenio) y que usará biodiesel como su combustible, por último, con los resultados se procedió al modelamiento con ayuda de la herramienta informática (SimaPro) y así realizar el ACV o la determinación de los impactos que generará este automotor.

\subsection{Obtención de la información}

Se extrae la información específica de todas las fases, tanto las previas, como son las técnicas agrarias para el sembrado y posterior recolección de las semillas, siguiendo con el procesamiento del aceite extraido hasta su transformación en biodiesel, usando la bibliografía relacionada y los artículos que del tema se han elaborado para la producción de biocombustibles, además por la información suministrada por una empresa nacional de procesamiento de aceite de palma. Se estableció las cantidades de insumos necesarios en cada proceso involucrado con el fin de obtener un producto final, de acuerdo con los datos de producción, diagramas de flujo (entradas y salidas) de materias primas y subproductos; en el caso de uso de fuentes energéticas 
eléctricas y calor se recurrió a los datos suministrados por la empresa, así como el uso del suelo y los niveles de fijación de $\mathrm{CO}_{2}$ tomado por los cultivos de palma africana en el proceso de fotosíntesis presente en las plantaciones fueron tomados de la base de datos de Ecoinvent (base de datos que suministra el software
SimaPro), otros datos están sustentados en el estudio estadístico para todas las plantaciones de palma africana en todo el territorio de los Llanos Orientales del país ya que este es el sector de mayor producción de cultivos de palma africana con aproximadamente 141.068 ha de cultivo (Fedepalma, 2015).

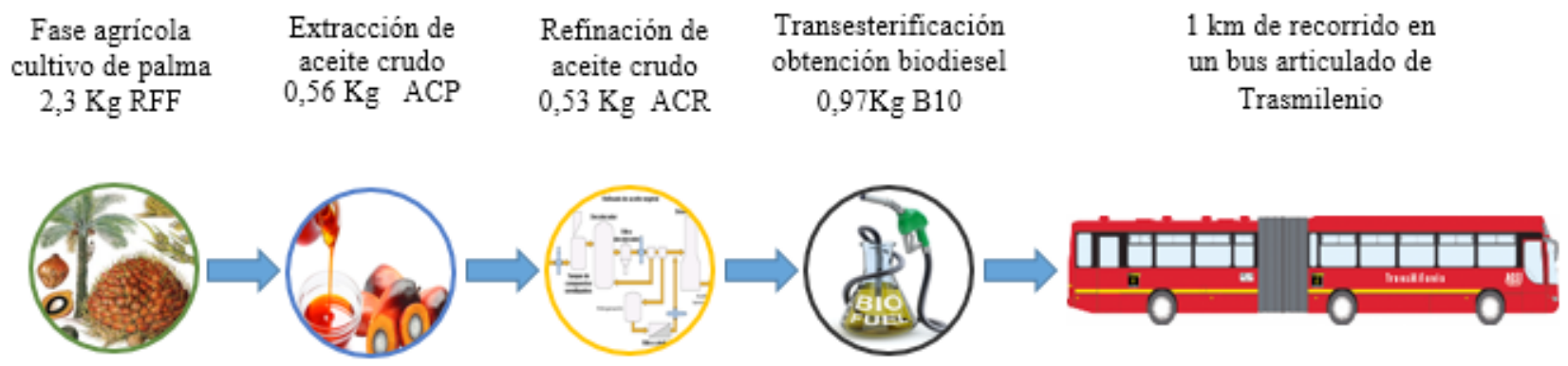

Fig. 1. Unidades funcionales vinculadas en el recorrido de un kilómetro de un bus articulado.

En la Figura 1 se observan las cantidades necesarias de los productos finales de cada subproceso, con el fin de satisfacer $1 \mathrm{~km}$ de trayecto en un bus articulado. El sistema abarca toda la fase agrícola (plantaciones de palma africana), que comprende un producto final que son los racimos de fruta fresca, posterior a esto se procede a la extracción de aceite crudo de palma en donde se observan procesos industriales como son: esterilización, digestión, prensado y clarificación, luego de obtener el aceite crudo se inicia el proceso de refinación del mismo, este es un proceso de acondicionamiento con el fin de remover las impurezas. Por último, se presenta la fase de transesterificación, el cual es un proceso fisicoquímico en donde se presentan reacciones químicas que dan lugar a la obtención de metilester (biodiesel), este proceso se caracteriza por la reacción del aceite vegetal con un alcohol (metanol) y la presencia de un catalizador, por lo general hidróxido de sodio; en el proceso también se genera glicerina que es una materia prima utilizada en la industria farmacéutica (Torres, 2012).

\subsection{Balances másicos y energéticos}

Se reconocieron las materias primas y consumos energéticos utilizados en las etapas que comprenden la producción del biodiesel y así mismo los subproductos originados por estas actividades (Torres, Contento \& Herrera, 2017).

\subsection{Análisis de ciclo de vida}

Se desarrollaron cuatro etapas que abarca el ACV sugeridas por la norma técnica internacional ISO 14044.

\subsubsection{Definición del objetivo y alcance del estudio}

De acuerdo con la metodología el primer paso para el desarrollo del ACV es la definición de los objetivos del estudio, así mismo se especifica la información que se espera obtener como resultado. En la Figura 1 se presentan en forma esquemática los límites del sistema para un estudio "cradle to gate". El alcance examina desde la adquisición de los distintos insumos u materias primas utilizadas en cada una de las fases del proceso productivo de obtención del biocombustible: plantación de palma africana, extracción de aceite de palma y su posterior transformación del aceite de palma a biodiesel. Así mismo, no se tienen en cuenta los siguientes aspectos: distribución y transporte, traslado del producto final al cliente, mantenimiento de maquinaria y equipos, gestión de los residuos, cargas a mbientales generados por la utilización del producto final, en este caso biocombustibles (emisiones atmosféricas) (Torres \& Herrera, 2017). 


\subsubsection{Análisis de inventario}

Se definieron los flujos de entrada y salida de materiales y energía, se calcularon las demandas de materias primas para la generación de biodiesel proveniente de aceite crudo de palma africana, y las emisiones ocasionadas a partir de estas actividades, así como las sustancias contaminantes producidas.

\subsubsection{Evaluación de impacto}

Se seleccionó el método de cálculo Traci 2.1, el cual es una herramienta que ha sido desarrollada para la sostenibilidad, evaluación del impacto del ciclo de vida, ecología industrial e impacto del diseño del proceso, evaluación para desarrollar productos, procesos, instalaciones, empresas y comunidades cada vez más sostenibles. Traci 2.1 esta incorporado en el software SimaPro, a su vez analiza las categorías de impacto: Aagotamiento del ozono, calentamiento global, acidificación, eutrofización, carcinógenos, no carcinógenos, efectos respiratorios, ecotoxicidad (Bare, Young \& Hopton, 2012).

\subsubsection{Interpretacion de resultados}

Una vez identificadas las materias primas e insumos que ocasionan los impactos más relevantes para cada proceso, la interpretación de análisis de resultados favoreció proponer las conclusiones de acuerdo con la magnitud de afectación medioambiental y la formulación de sugerencias con el fin instaurar mejoras en los procesos de producción y de esta manera poder mitigar, controlar y minimizar las cargas ambientales ocasionadas por dichas actividades.

\subsection{Herramientas informáticas utilizadas}

Por otra parte, para la evaluación de los impactos del ciclo de vida, se usó la herramienta informática comercial SimaPro 8.1 (http://www.pre.nl/simapro). SimaPro es una herramienta desarrollada por Pré Consultants, y permiteanalizar y comparar los aspectos medioambientales de un producto o proceso de una manera sistemática y consistente, cumpliendo las recomendaciones de las normas ISO 14040 e ISO 14044. El software cuenta con la base de datos Ecoinvent que proporciona datos a nivel mundial sobre una gran variedad de procesos. Una vez desarrollados los inventarios de las diferentes etapas del proceso, esta información se ingresó en el software y se obtuvieron los datos de los impactos evaluados, a partir de los factores de caracterización de las corrientes identificadas en el inventario.

\section{Resultados}

A continuación, se presentan de manera específica los inventarios de cada una de las etapas del proceso, y las cargas ambientales generadas según las categorías de impacto evaluadas (Franco \& Ordońez, 2019).

\subsection{Analisis por procesos}

Los resultados del análisis de inventario contienen la información detallada de las entradas y salidas de materia y energía de cada uno de los cuatros subprocesos evaluados. En la Tabla 1 se observa el conjunto de datos que representa la producción de racimos de fruta fresca de palma en Colombia. Todos los intercambios se calculan por $2,3 \mathrm{~kg}$ de racimo de fruta fresca (masa húmeda) y se basan en datos de campo promedio para áreas de cultivo industrial de palma aceitera en Colombia; adicionalmente, los insumos agrícolas fueron registrados con las características generales de materia prima consumida, tales como cantidad y composición química. En la Tabla 2, se observa los flujos de entradas y salidas para el proceso de separación de aceite crudo de palma de acuerdo con los datos obtenidos en la extractora, estos datos satisfacen una producción de $0,56 \mathrm{Kg}$ de aceite crudo de palma, se observan algunos agentes químicos y las cantidades de energía utilizadas. 


\section{Tabla 1}

Entradas y salidas proceso de produccion de racimos de frutra freca

\begin{tabular}{|c|c|c|}
\hline \multicolumn{3}{|c|}{ Inventario producción de racimo de fruta fresca } \\
\hline \multicolumn{3}{|c|}{ Salidas o productos } \\
\hline Producto & Cantidad & Unidad \\
\hline Racimo de fruta fresca & 2,3 & $\mathrm{~kg}$ \\
\hline \multicolumn{3}{|c|}{ Entradas desde la naturaleza } \\
\hline Producto & Cantidad & Unidad \\
\hline Dióxido de carbono $\left(\mathrm{CO}_{2}\right)$ & 3,38 & $\mathrm{~kg}$ \\
\hline Poder calorífico & 36,8 & MJ \\
\hline Ocupación bosque intensivo & 1,21 & $\mathrm{~m}^{2}$ \\
\hline Transformación, a partir de cultivo & 0,02 & $\mathrm{~m}^{2}$ \\
\hline \multicolumn{3}{|c|}{ Entradas conocidas materiales o combustibles } \\
\hline Producto & Cantidad & Unidad \\
\hline Sulfonilurea & 16,65 & $\mathrm{~kg}$ \\
\hline Tiocarbamato & 3,7 & $\mathrm{~kg}$ \\
\hline Acetamida-anilida & 7,82 & $\mathrm{~kg}$ \\
\hline Nitrato de amonio & $2,3 \times 10^{-3}$ & $\mathrm{~kg}$ \\
\hline Riego & 0,023 & \\
\hline Cambio de uso del suelo, cultivo perenne, de uso perenne & $5 \times 10^{-5}$ & ha \\
\hline Oxido de magnesio & $1,8 \times 10^{-3}$ & $\mathrm{~kg}$ \\
\hline Fertilizante nitrogenado & $2 \times 10^{-3}$ & $\mathrm{~kg}$ \\
\hline Compuestos organofosforados & 6,5 & $\mathrm{~kg}$ \\
\hline Pesticidas no especificados & 4,23 & $\mathrm{~kg}$ \\
\hline Fertilizante de fosfato & $4,6 \times 10^{-3}$ & $\mathrm{~kg}$ \\
\hline Ftalamida & 6,12 & $\mathrm{~kg}$ \\
\hline Urea, como n & $4,6 \times 10^{-3}$ & $\mathrm{~kg}$ \\
\hline Transporte, carga, camión 16-32 toneladas métricas, euro3 & 0,02 & $\mathrm{tkm}$ \\
\hline Transporte, coche de pasajeros, tamaño grande, gasolina, euro3 & $1 \times 10^{-3}$ & $\mathrm{~km}$ \\
\hline transporte, tractor y remolque & 0,02 & $\mathrm{tkm}$ \\
\hline \multicolumn{3}{|c|}{ Salidas o subproductos } \\
\hline \multicolumn{3}{|c|}{ Emisiones al agua } \\
\hline \multicolumn{3}{|l|}{ Producto } \\
\hline Nitrato & 0,02 & $\mathrm{~kg}$ \\
\hline Fosfato & $2 \times 10^{-5}$ & $\mathrm{~kg}$ \\
\hline Fósforo & $4 \times 10^{-4}$ & $\mathrm{~kg}$ \\
\hline Agua & 0,01 & $\mathrm{~m}^{3}$ \\
\hline Agua & $2 \times 10^{-3}$ & $\mathrm{~m}^{3}$ \\
\hline \multicolumn{3}{|c|}{ Emisiones al suelo } \\
\hline Producto & Cantidad & Unidad \\
\hline Azoxistrobina & 20,06 & $\mathrm{~kg}$ \\
\hline Cadmio & 22,45 & $\mathrm{~kg}$ \\
\hline Carbendazim & 13,41 & $\mathrm{~kg}$ \\
\hline Carbofuran & 3,66 & $\mathrm{~kg}$ \\
\hline Cromo & 4,95 & $\mathrm{~kg}$ \\
\hline Diquat & 6,12 & $\mathrm{~kg}$ \\
\hline Glifosato & $3 \times 10^{-4}$ & $\mathrm{~kg}$ \\
\hline
\end{tabular}


TABla 2

Entrada y salidas del proceso de extracción de aceite crudo de palma africana

\begin{tabular}{|c|c|c|}
\hline \multicolumn{3}{|c|}{ Inventario de la fase extracción de aceite crudo } \\
\hline \multicolumn{3}{|c|}{ Entradas conocidas (materiales/combustibles) } \\
\hline Producto & Cantidad & Unidad \\
\hline Amoniaco, líquido & $12 \times 10^{-7}$ & $\mathrm{~kg}$ \\
\hline Químicos, orgánico & $8 \times 10^{-5}$ & $\mathrm{~kg}$ \\
\hline Cloro, gaseoso & $5 \times 10^{-6}$ & $\mathrm{~kg}$ \\
\hline Unidad de cogeneración de calor y energía, $6400 \mathrm{kw}$ & $3 \times 10^{-9}$ & $\mathrm{p}$ \\
\hline Hexano & $3 \times 10^{-3}$ & $\mathrm{~kg}$ \\
\hline Aceite lubricante & $5 \times 10^{-5}$ & $\mathrm{~kg}$ \\
\hline Molino de aceite & $1 \mathrm{X} 10^{-9}$ & $\mathrm{p}$ \\
\hline Racimo de fruta fresca & 2,3 & $\mathrm{~kg}$ \\
\hline Ácido fosfórico, grado industrial, sin agua, en estado de solución al 85\% & $1 \mathrm{X} 10^{-4}$ & $\mathrm{~kg}$ \\
\hline Cloruro de sodio, polvo & $1 \mathrm{X} 10^{-4}$ & $\mathrm{~kg}$ \\
\hline Agua de grifo & 12,01 & $\mathrm{~kg}$ \\
\hline \multicolumn{3}{|c|}{ Salidas o subproductos } \\
\hline \multicolumn{3}{|c|}{ Emisiones al aire } \\
\hline Producto & Cantidad & Unidad \\
\hline Acetaldehido & $1 \times 10^{-5}$ & $\mathrm{~kg}$ \\
\hline Amoníaco & $21 \times 10^{-5}$ & $\mathrm{~kg}$ \\
\hline Arsénico & $1 \times 10^{-8}$ & $\mathrm{~kg}$ \\
\hline Benzeno & $1 \mathrm{X} 10^{-5}$ & $\mathrm{~kg}$ \\
\hline Bromo & $1 \times 10^{-5}$ & $\mathrm{~kg}$ \\
\hline Cadmio & $1 \times 10^{-8}$ & $\mathrm{~kg}$ \\
\hline Calcio & $1 \times 10^{-4}$ & $\mathrm{~kg}$ \\
\hline Dióxido de carbono,biogénico & 1,82 & $\mathrm{~kg}$ \\
\hline Monóxido de carbono, biogénico & $1 \times 10^{-4}$ & $\mathrm{~kg}$ \\
\hline Cloro & $3 \times 10^{-6}$ & $\mathrm{~kg}$ \\
\hline Óxido de nitrógeno & $1 \times 10^{-3}$ & $\mathrm{~kg}$ \\
\hline Compuestos orgánicos volátiles no metálicos & $1 \times 10^{-5}$ & $\mathrm{~kg}$ \\
\hline Hidrocarburos aromáticos policíclicos & $2 \times 10^{-7}$ & $\mathrm{~kg}$ \\
\hline Partículas, $<2.5$ um & $1 \times 10^{-3}$ & $\mathrm{~kg}$ \\
\hline Dióxido de azufre & $4 \times 10^{-5}$ & $\mathrm{~kg}$ \\
\hline Tolueno & $4 \times 10^{-6}$ & $\mathrm{~kg}$ \\
\hline Agua $/ \mathrm{m}^{3}$ & $2 \times 10^{-3}$ & $\mathrm{~m}^{3}$ \\
\hline Zinc & $4 \times 10^{-6}$ & $\mathrm{~kg}$ \\
\hline \multicolumn{3}{|c|}{ Salidas o subproductos } \\
\hline \multicolumn{3}{|c|}{ Emisiones al agua } \\
\hline Producto & Cantidad & Unidad \\
\hline Agua & 0,01 & $\mathrm{~m}^{3}$ \\
\hline \multicolumn{3}{|c|}{ Salidas o subproductos } \\
\hline \multicolumn{3}{|c|}{ Residuos y emisiones para tratamiento } \\
\hline Producto & Cantidad & Unidad \\
\hline Residuos de aceite mineral & $5 \times 10^{-5}$ & $\mathrm{~kg}$ \\
\hline Aguas residuales residenciales & $3 \times 10^{-5}$ & $\mathrm{~m}^{3}$ \\
\hline Mezcla de ceniza de madera, pura & $7 \times 10^{-3}$ & $\mathrm{~kg}$ \\
\hline
\end{tabular}


En la Tabla 3 se presentan las entradas y salidas de materia y energía calculadas para el proceso de refinamiento de aceite crudo de palma. Las materias primas utilizadas se caracterizan para: electricidad de media tensión, calor a partir de gas natural y un agente químico como es el hidróxido de sodio de solución al 50 \%.

\section{TABLA 3}

Entradas y salidas proceso de refinacion de aceite crudo de palma africana

\begin{tabular}{|c|c|c|}
\hline \multicolumn{3}{|c|}{ Inventario de la fase de refinación de aceite crudo } \\
\hline \multicolumn{3}{|c|}{ Salidas o productos } \\
\hline Producto & Cantidad & Unidad \\
\hline Aceite de palma, refinado & 0,53 & $\mathrm{~kg}$ \\
\hline \multicolumn{3}{|c|}{ Entradas conocidas (materiales/combustibles) } \\
\hline Producto & Cantidad & Unidad \\
\hline Electricidad, media tensión & $4 \times 10^{-3}$ & kwh \\
\hline $\begin{array}{l}\text { Calor, distrito o industrial, gas } \\
\text { natural }\end{array}$ & 0,06 & MJ \\
\hline Aceite de palma crudo & 1,042 & $\mathrm{~kg}$ \\
\hline Jabón & $-74 \times 10^{-4}$ & $\mathrm{~kg}$ \\
\hline $\begin{array}{l}\text { Hidróxido de sodio, sin agua, } \\
\text { en estado de solución al } 50 \%\end{array}$ & $2,3 \times 10^{-3}$ & $\mathrm{~kg}$ \\
\hline Agua del grifo & 156 & $\mathrm{~kg}$ \\
\hline Refinería de aceite vegetal & $1 \mathrm{X} 10^{-10}$ & $\mathrm{p}$ \\
\hline \multicolumn{3}{|c|}{ Salidas o subproductos } \\
\hline \multicolumn{3}{|c|}{ Emisiones al aire } \\
\hline Producto & Cantidad & Unidad \\
\hline Agua $/ \mathrm{m}^{3}$ & 0,07 & $\mathrm{~m}^{3}$ \\
\hline \multicolumn{3}{|c|}{ Salidas o subproductos } \\
\hline \multicolumn{3}{|c|}{ Residuos y emisiones para tratamiento } \\
\hline Producto & Cantidad & Unidad \\
\hline Aguas residuales de refinería & 0,09 & $\mathrm{~m}^{3}$ \\
\hline
\end{tabular}

Según las unidades funcionales parciales basadas en el cálculo del Biodiesel (B10) requerido para poder lograr un kilómetro de trayecto en un bus articulado, se encontró que la cantidad necesaria para cumplir tal función es $0,97 \mathrm{Kg}$ (B10), por ende, todos los flujos (entradas y salidas) del proceso de transesterificación estarán soportados bajo esta cantidad, es decir se estimaran las cantidades netas de materias primas y subproductos que suplan $0,97 \mathrm{Kg}$ (B10) como se aprecia en la Tabla 4.

\section{TABLA 4}

Datos para la transesterificación u obtención de biodiesel

\begin{tabular}{|c|c|c|}
\hline \multicolumn{3}{|c|}{ Inventario de la fase de producción de biodiesel } \\
\hline \multicolumn{3}{|c|}{ Salidas o productos } \\
\hline Producto & Cantidad & Unidad \\
\hline Biodiesel, b10 & 0,97 & $\mathrm{~kg}$ \\
\hline \multicolumn{3}{|c|}{ Entradas desde la naturaleza } \\
\hline Producto & Cantidad & Unidad \\
\hline Dioxido de carbono $\left(\mathrm{Co}_{2}\right)$ & 0,21 & $\mathrm{~kg}$ \\
\hline Agua, refrigeración, & $3 \times 10^{-4}$ & $\mathrm{~m}^{3}$ \\
\hline \multicolumn{3}{|c|}{ Entradas conocidas (materiales/combustibles) } \\
\hline Producto & Cantidad & Unidad \\
\hline Electricidad, media tensión & 0,0388 & $\mathrm{kWh}$ \\
\hline Glycerine & $-0,097$ & $\mathrm{~kg}$ \\
\hline Calor, industrial, gas natural & 0,89 & MJ \\
\hline Metanol & 0,1 & $\mathrm{~kg}$ \\
\hline Aceite crudo de palma & 0,56 & $\mathrm{~kg}$ \\
\hline Ácido fosfórico & $4 \times 10^{-3}$ & $\mathrm{~kg}$ \\
\hline Hidróxido de potasio & 0,01 & $\mathrm{~kg}$ \\
\hline Agua de grifo & 0,02 & $\mathrm{~kg}$ \\
\hline Planta de esterificación & 9,05 & $\mathrm{P}$ \\
\hline \multicolumn{3}{|c|}{ Salidas o subproductos } \\
\hline \multicolumn{3}{|c|}{ misiones al aire } \\
\hline Dióxido de carbono, biogénico & 0,29 & $\mathrm{~kg}$ \\
\hline \multicolumn{3}{|c|}{ misiones al agua } \\
\hline Agua residual & 4,2 & $\mathrm{~m}^{3}$ \\
\hline
\end{tabular}




\subsection{Evaluación de impacto}

A continuación, en las figuras 2 a 5 se presentan las cargas más significativas en los procesos para la obtención de biodiesel. Como se aprecia en las figuras 2 y 3, se encontró que las fases agrícolas y de extracción de aceite interfieren directamente con la categoría de ecotoxicidad, a su vez en estos dos subprocesos se encuentra que las materias primas que más afectan son los fertilizantes y pesticidas a base de agentes químicos como nitrógeno, fósforo y potasio. Se observa en la Figura 2 una contribución favorable al medio ambiente y se observa en la categoría de calentamiento global, esto se debe a que el cultivo de palma africana fija el $\mathrm{CO}_{2}$. Según un estudio de Fedepalma, realizado en el año 2016, un grupo de investigadores encontró que este cultivo captura 29 kilogramos de carbono por hectárea cada día, esto favorece a subsanar las emisiones resultantes del proceso productivo.

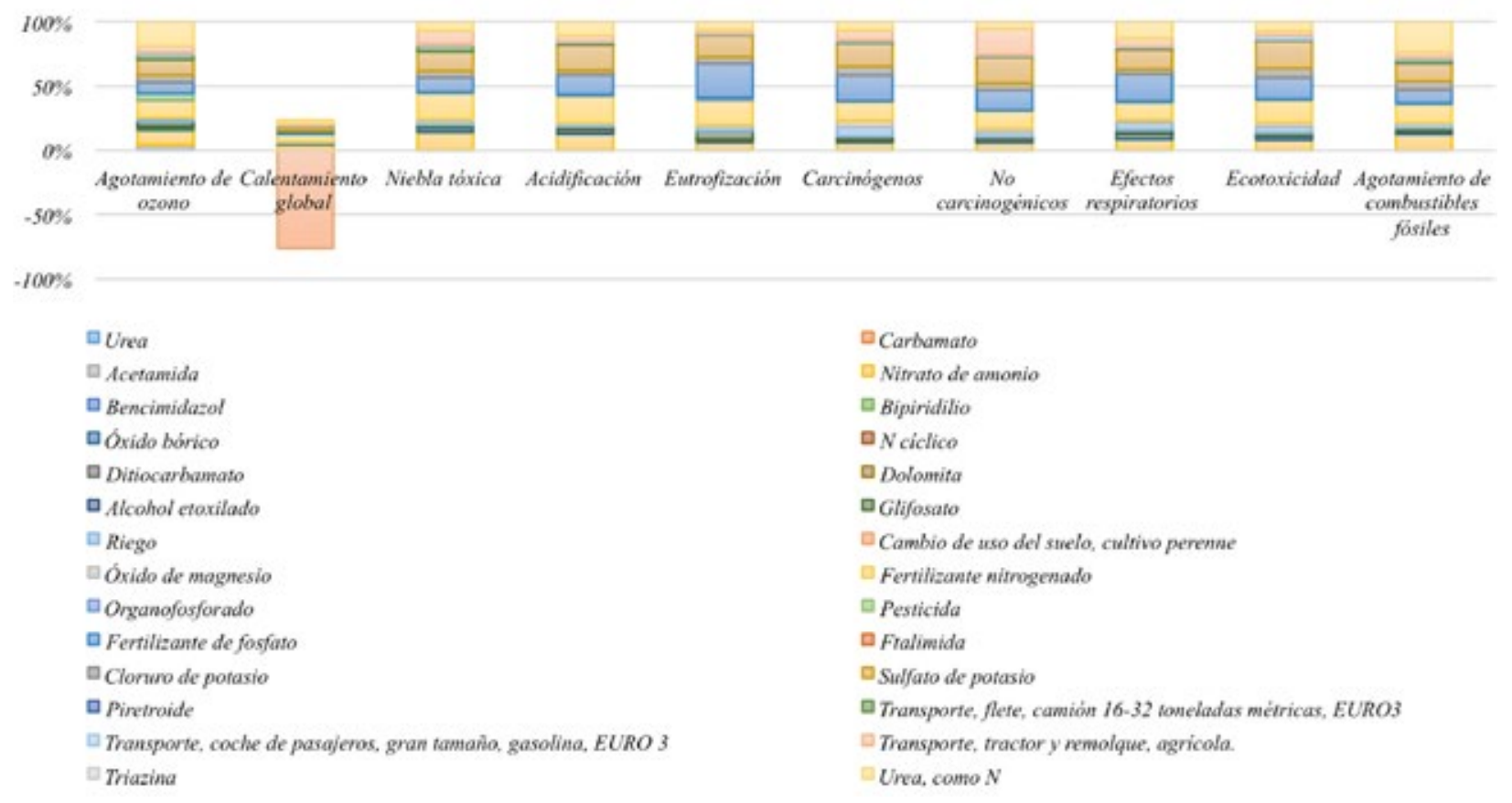

Fig. 2 Perfil ambiental proceso agrícola en la obtención de racimos de fruta fresca

En el proceso de extracción de racimos de fruta fresca también es posible observar cargas ambientales favorables, exactamente en la categoría de Efectos no cancerígenos, por lo que se concluye que el impacto positivo en esta categoría se genera debido a la fijación de $\mathrm{CO}_{2}$ por parte del cultivo de palma africana, la cual es vital para su fotosíntesis, estas emisiones son llevadas a cabo por los vehículos y maquinaria agrícola utilizada.

En el caso del proceso de refinación, teniendo en cuenta su perfil ambiental presentado en la Figura 4, se evidencia que los insumos que se reportan con mayor frecuencia en las diferentes categorías son el agua potable, la generación de aguas residuales y por supuesto el principal insumo que es el aceite crudo de palma, encontrándose una incidencia en todas las categorías principalmente en el calentamiento global, acidificación, eutrofización, y ecotoxicidad, aunque también es posible observar contribuciones favorables, y esto se debe principalmente a el reuso de materias primas como el gas y el jabón, lo que genera estos impactos favorables sobre el medio ambiente dado que se está evitando el vertimiento de sustancias líquidas por esta actividad industrial. 


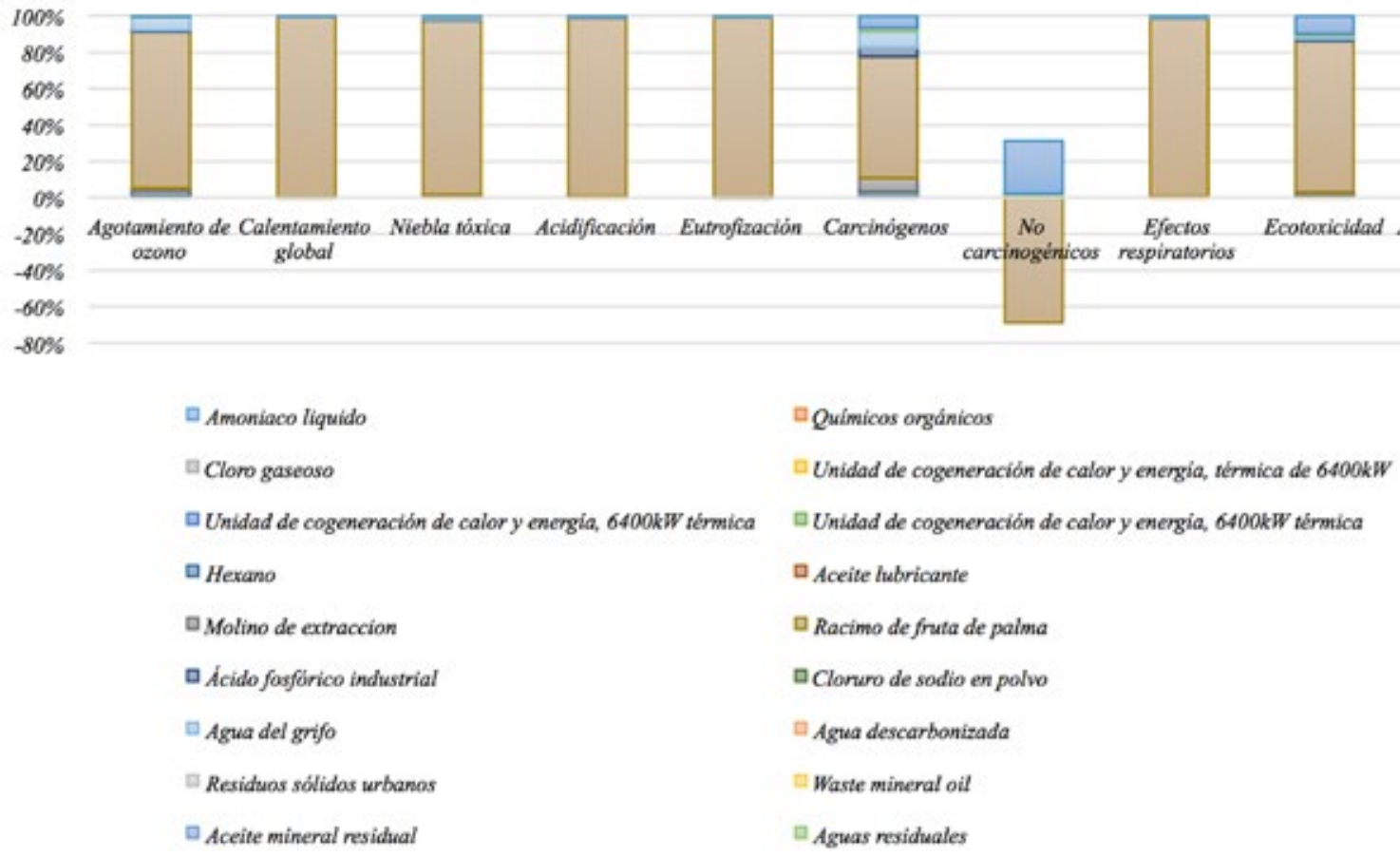

Fig. 3. Perfil ambiental proceso de extracción de aceite crudo de palma africana

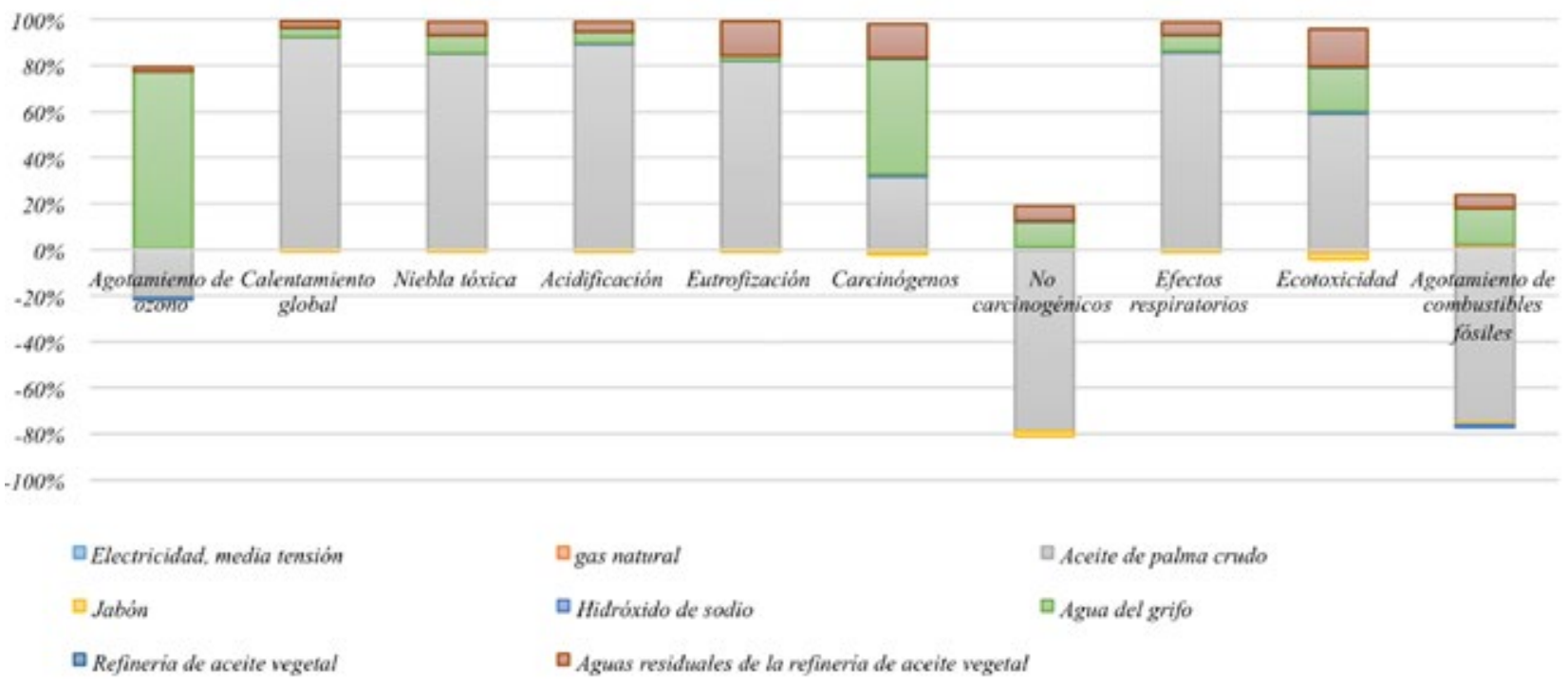

Fig. 4. Perfil ambiental fase de refinación de aceite crudo de palma africana

Cabe resaltar que los principales efectos positivos, en la fase de refinación de aceite crudo, se deben a la reutilización de materias primas como el jabón, por ende se contemplan las posibilidades de reducir los principales efectos negativos en categorías como Agotamiento de ozono, Efectos no cancerigenos y
Agotamiento de combustibles fosiles. Como el proceso de refinación se caracteriza por ser un proceso industrial donde es necesario la utilización de agentes químicos y energía (gas y electricidad), por ende se pueden contribuir las afectaciones ambientales a estas materias primas. 
El último proceso en la obtención de biodiesel corresponde a la transesterificación, como se evidencia en la Figura 5, que las materias primas que tienen mayor carga ambiental son el aceite crudo de palma, la glicerina y por último el metanol; cabe resaltar que estas tres materias primas mencionadas son fundamentales en el proceso de transesterificación, así mismo se observa que las contribuciones favorables al medio ambiente se dan en las siguientes categorías: Agotamiento de ozono (glicerina y aceite crudo de palma), Calentamiento global (glicerina), Cancerígenos (gas natural y metanol), No cancerígenos (aceite crudo de palma, gas natural y metanol), Efectos respiratorios (gas natural y metanol), Ecotoxicidad (gas natural y metanol), Agotamiento de combustibles fósiles (glicerina y aceite crudo de palma).

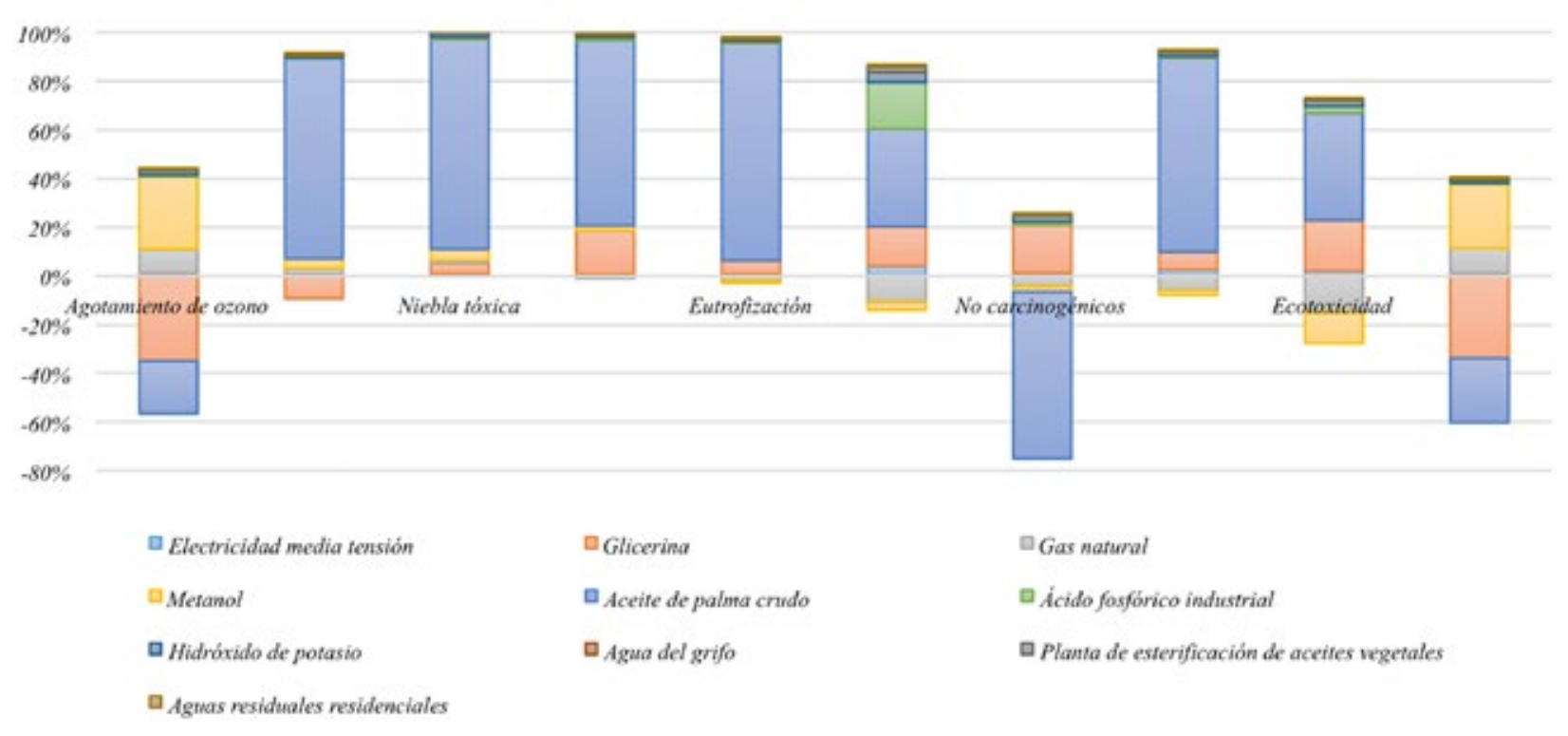

Fig. 5 Perfil ambiental fase transesterificación u obtención de biodiesel

Las cargas favorables al medio ambiente se producen por la recirculación de insumos como el metanol y el gas, así mismo, en la fase de transesterificación se genera un subproducto originado del proceso químico (reacción entre el aceite crudo de palma, metanol y el catalizador) que da como resultado la glicerina, este subproducto es utilizado en la industria farmacéutica, de este modo se está evitando un residuo, razón por la cual se generan las contribuciones favorables.

Para finalizar, las contribuciones positivas en el proceso de transesterfiacion se deben a que los subproductos generados en este proceso se utilizan como materias primas en otras industrias, como es el caso de la glicerina.

\subsection{Comparación entre procesos}

En la Figura 6 se muestra el perfil medio ambiental consecuencia de la equiparación entre las fases (producción agrícola de racimos de fruta fresca, extracción de aceite crudo de palma, refinación de aceite crudo de palma y transesterificación). En consecuencia, se recomienda plantear ideas de optimización en los distintos procesos estudiados como son planes de manejo ambiental que permitan establecer acciones que prevengan, controlen y corrijan los posibles efectos provocados en la obtención del biodiesel derivado de la palma de aceite, que incluya planes de seguimiento, evaluación y monitoreo y las acciones de contingencia en caso de algún percance (Díaz, 2012; Vidal, Quintero \& Herrera, 2017; Pinzón \& Palacios, 2016). 


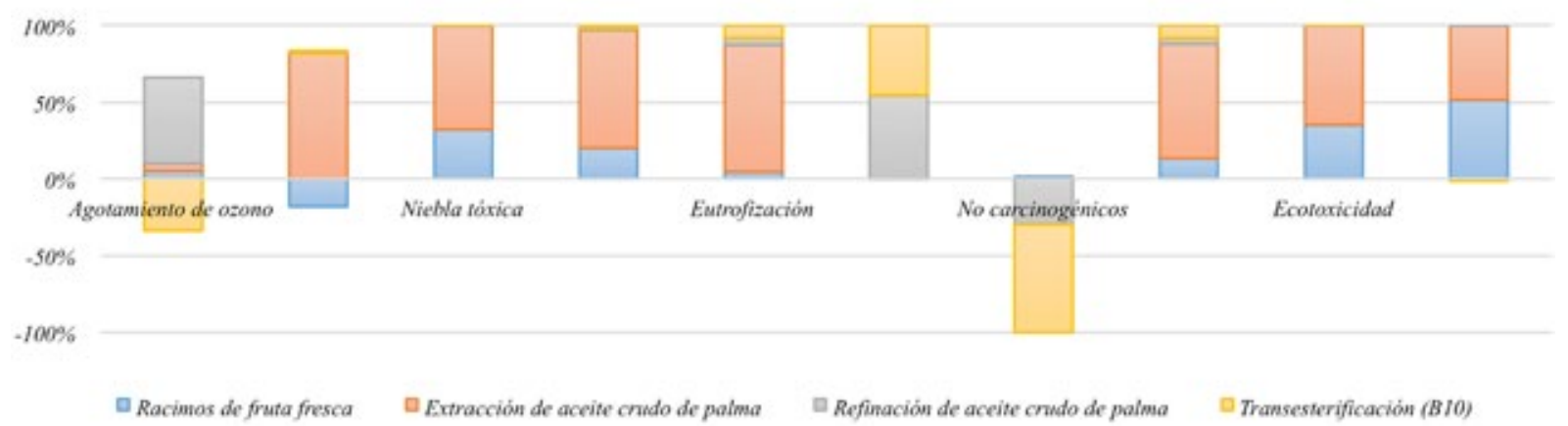

Fig. 6 Perfil ambiental para la comparación de los procesos involucrados en la obtención de (B10)

De esta manera es posible constatar que el proceso que más incidencia tiene sobre todas las categorías de impacto, es el proceso de extracción de aceite crudo de palma, como se observa en la Figura 6, que básicamente se debe al uso de los recursos naturales para el funcionamiento de la planta extractora, específicamente el agua, dado que este recurso es esencial para el proceso de extracción de aceite por la alta utilización de vapor de aire caliente, el consumo de combustible y la energía eléctrica, todos involucrados con las actividades de la planta. De otro lado las emisiones de gases de efecto invernadero en las labores de extracción, es una de las cargas ambientales más representativas como el óxido Nitroso $\left(\mathrm{NO}_{\mathrm{x}}\right)$ y el material particulado liberado en las calderas de la planta extractora.

El segundo proceso que más aporta a las cargas ambientales es la actividad agrícola, la recolección de los racimos de fruta fresca afecta consideradamente la categoría de ecotoxicidad. Examinando dicho proceso, se puede deducir que los insumos que mayor incidencia tuvieron fueron los fertilizantes a base de potasio, fosfato, nitrógeno y los pesticidas, este comportamiento de debe a aspectos como la dosis empleada, el estado de desarrollo del cultivo, los niveles máximos tolerables del fruto, tiempos de residencia y otras propiedades fisicoquímicas; el alto grado de los plaguicidas enmascara el efecto de las emisiones por metales pesados como el plomo y el cromo.

El tercer proceso con mayor significancia ambiental es la transesterificación u obtención del biodiesel, dado que es un proceso netamente industrial, por ende se originan emisiones atmosféricas y vertimientos. Cabe resaltar que en este proceso se generan residuos que posteriormente se utilizan como materias primas, como el caso de la glicerina, es decir se evita un residuo, esto se ve claramente reflejado en la Figura 6 en las contribuciones positivas.

Por último y no menos importante, el proceso de refinamiento de aceite crudo es la que menos incidencia tiene sobre las diferentes categorías de impacto, dado que requiere de pocas materias primas y algunas se recirculan.

\section{Conclusiones}

En la determinación de las cargas ambientales más representativas durante el proceso de obtención de biocombustible, se encontraron impactos positivos como negativos que se mencionan a continuación:

\section{Impactos positivos}

Cambio climático y agotamiento de la capa de ozono derivados del proceso agrícola: producción de racimos de fruta fresca debido a la captura de $\mathrm{CO}_{2}$.

Agotamiento de combustibles fósiles No cancerígenos, reducción del Agotamiento del ozono y la Ecotoxicidad debidos recirculamiento de algunas materias primas como el metanol y al reusó de la glicerina entre otros.

\section{Impactos negativos}

Se pudo observar que la categoría más impactada en 
los procesos involucrados en la obtención de biodiesel fue la ecotoxicidad, presente en las fases de producción agrícola de racimos de frutos frescos y la extracción de aceite crudo de palma, generados por la utilización de pesticidas y fertilizantes a base de nitrógeno, potasio y fosfatos.

En los dos procesos faltantes (refinación y transesterificación) se evidenció que la categoría más impactada es la de efectos cancerígenos, esto es debido al uso y exposición de agentes químicos particularmente para uso industrial.

De acuerdo con las distintas categorías de impacto que se evaluaron usando el software SimaPro, se concluye que la extracción de aceite crudo de palma africana es la actividad de mayor impacto ambiental. Una vez reconocidas las cargas ambientales que genera la producción de biodiesel es indispensable establecer una toma de decisiones que impliquen a la disminución de los mismos mediante planes de manejo ambiental para los distintos procesos involucrados en la producción de biodiesel (B10) y darle un valor agregado a los coproductos generados (Tan et al., 2016; Martínez, 2017).

\section{Agradecimientos}

Agradecemos a la empresa BioD S.A. por su respaldo y apoyo para el desarrollo del presente proyecto. Igualmente, a la Federación Colombiana de Biocombustibles - Fedebiocombustibles por los datos brindados. Especial reconocimiento al Centro de Investigaciones Energéticos Medioambientales y Tecnológicos - Ciemat por la capacitación brindada.

\section{REFERENCIAS}

Arango, S. \& Torres A. (2008). Incidencias económicas del etanol como combustible en Colombia sobre los derivados de la cańa de azúcar: una aproximación con dinámicas de sistemas. Revista Avances en Sistemas e Informática, 5(2), 69-75.

Bare, J., Young, D. \& Hopton, M. (2012). Tool for the Reduction and Assessment of Chemical and other Environmental Impacts
(TRACI) - User's Manual. United States Environmental Protection Agency (EPA), ORD/NRMRL/Sustainable Technology Division/ Systems Analysis Branch. Cincinnati, Ohio 45268: Standard Operating Procedure.

Díaz Y. (2012). Impacto ambiental generado a partir de biodiesel de aceite de palma. Tesis. Bogotá: Universidad Libre.

Fedepalma (2015). The Oil Palm Agroindustry in Colombia and World 2010 - 2014. Statistical Yearbook 2015. Bogotá: Federación Nacional de Cultivadores de Palma de Aceite - Fedepalma.

Franco, J. \& Ordóńez, L. (2019). Análisis del ciclo de vida para la producción de biodiesel a partir de aceite de la palma africana usando la metodología ISO 14044 para la empresa BIOD S.A. (Facatativá - Cundinamarca). Tesis. Bogotá: Universidad de La Salle.

Huergo E. (2018). Santa Fe (Argentina) utilizará biodiesel puro en el transporte público de toda la provincia. Biocombustibles Hoy, Boletín No. 192. Federación Colombiana de Biocombustibles - Fedebiocombustibles. Recuperado en: http://www.fedebiocombustibles.com/nota-web-id-3095.htm

Martínez D. (2017). Evaluación de la sostenibilidad de tecnologías emergentes de producción de biocombustibles en Colombia aplicando criterios técnicos, ambientales y económicos. Tesis. Bucaramanga: Universidad Industrial de Santander.

McDonough, W., Braungart, M. (2002). Cradle to Cradle. New York: North Point Press.

Ministerio de Minas y Energía (2018). Resolución 40184 (febrero 27 del 2018). Por la cual se modifica la Resolución número 182142 de 2007, en relación con el porcentaje de mezcla de biocombustible para uso en motores diésel, en Bogotá, D. C., centro del país y Llanos Orientales. República de Colombia. Recuperado de: http://201.217.193.253/labpalma/docs/pdf/ resolucion_minminas_40184_2018.pdf

Pinzón, W. \& Palacios, D. (2016). Determinación de la huella hídrica en el cultivo de palma de aceite en la empresa Guaicaramo S.A. localizada en Barranca de Upía-Meta. Tesis. Bogotá: Universidad de La Salle.

Tan, J., Jahim, J., Harum, S., Wu, T. \& Mumtaz, T. (2016). Utilization of oil palm fronds as a sustainable carbon source in biorefineries. International Journal of Hydrogen Energy, 41, 4896-4906.

Torres, J. (2012). Sulfonation/Sulfation Processing Technology for Anionic Surfactant Manufacture. Recuperado de: https://www. intechopen.com/books/advances-in-chemical-engineering/ sulfonation-sulfation-processing-technology-for-anionic-surfactant-manufacture. DOI: 10.5772/32077.

Torres, J., Contento, O. \& Herrera, I. (2017). Análisis de ciclo de vida para una biorefinería derivada de residuos agrícolas de palma aceitera (Elaeis guineensis). Publicaciones e Investigación, 11(1), 13-36.

Torres, J., Herrera, I., Garrain, D. \& Gamarra, A. (2018). Integration of Colombian biorefinery from industrial palm oil waste into the circular economy. Abstract Book SETAC Europe 28th Annual Meeting, 13-17 May 2018. Roma: Italia. 
Torres, J. \& Herrera, I. (2017). Modelo de cálculo para ACV de un cultivo de palma africana. En: Energias renovable y materiales para nuevas tecnologias en Colombia. Varios Autores. Bucaramanga: Ediciones UIS.

Torres, J., Morales, G., Suárez, O. \& Sánchez F. (2009). Mathematical model of a falling film reactor for methyl ester sulfo- nation. Chemical Product and Process Modeling, 4(5), DOI: 10.2202/1934-2659.1393.

Vidal, A., Quintero, J. \& Herrera, I. (2017). Análisis de ciclo de vida de la producción de biodiesel a partir de aceite vegetal usado, DYNA, 84(201), 155-162. 
Revista Especializada en Tecnología e Ingeniería 
